$\begin{array}{ll}\text { Research Square } & \begin{array}{l}\text { Preprints are preliminary reports that have not undergone peer review. } \\ \text { They should not be considered conclusive, used to inform clinical practice, } \\ \text { or referenced by the media as validated information. }\end{array}\end{array}$

\title{
The Response of Hypericum Perfpratum L. to the Application of Selenium and Nano-selenium
}

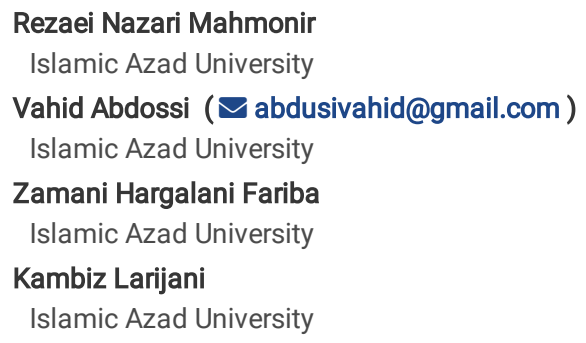

\section{Research article}

Keywords: Hypericum perforatum, hypericin, hyperforin, selenium

Posted Date: July 14th, 2021

DOI: https://doi.org/10.21203/rs.3.rs-708123/v1

License: (c) (i) This work is licensed under a Creative Commons Attribution 4.0 International License. Read Full License 


\section{Abstract}

Background: In terms of medicinal and therapeutic properties, H. perforatum is the important species. However, Selenium has been applied as an alleviation strategy subjected to producing essential oils and biomass.

Method: For this study, a randomized complete block design with three replications was used so that each experimental unit comprised of 30 number 8 pots. The treatments included the foliar application of selenium $(6,8,10$, and $12 \mathrm{mg} / \mathrm{l})$, nano-selenium $(6,8,10$, and $12 \mathrm{mg} / \mathrm{l})$, and control (distilled water), applied at the rosette stage and harvesting at $50 \%$ flowering stage.

Results: The results of the analysis of variance showed that the effect of selenium and nano selenium fertilizers was significant $(p<0.01)$ on plant height, inflorescence length, number of inflorescences, inflorescence weight, shoot weight, root length, the total weight of biomass, essential oil percentage, the content of hypericin and hyperforin, the selenium accumulation in the plant, relative leaf water content, the content of chlorophylls a, b, and total, the content of phenol, the content of proline, production and accumulation of catalase, peroxidase, malondialdehyde, and DPPH enzymes. The highest inflorescence weight $(21 \mathrm{~g} / \mathrm{plant})$, shoot weight $(70 \mathrm{~g} /$ plant), and essential oil content $(0.14 \%)$ were obtained from the control treatment. The highest accumulation of hypericin (3.8 mg/g dry matter) was obtained from the foliar application of $8 \mathrm{mg} / \mathrm{l}$ selenium. The maximum accumulation of hyperforin ( $57 \mathrm{mg} / \mathrm{g}$ dry matter) was obtained from the foliar application of $10 \mathrm{mg} / \mathrm{l}$ selenium. The highest accumulation of selenium ( $287.52 \mu \mathrm{g} / \mathrm{g}$ dry matter) was obtained in the foliar application of $12 \mathrm{mg} / \mathrm{l}$ nano-selenium.

Conclusion: If the purpose of producing $H$. perforatum is to use the shoots and essential oils of the plant, then the use of selenium and nano-selenium is not recommended at all and should not be used. If the goal is to produce high hypericin, high hyperforin, and also the accumulation of selenium in the plant, the use of treatments of 6 and $8 \mathrm{mg} / \mathrm{l}$ of selenium and nano-selenium could be applied.

\section{Background}

Hypericum perforatum belongs to the Hypericaceae family and has more than 469 species in the world (Crockett, 2010), of which 17 (Mozaffarian, 1996 ) to 19 species (Zobayed et al., 2005) of this genus have been reported in Iran. St. John's wort is a perennial plant and the most important medicinal species of this genus. It is a branched plant with $40-80 \mathrm{~cm}$ high. The stems and branches are covered with broad leaves and soft margins, and the leaves are 1 to $3 \mathrm{~cm}$ long and 0.3 to $1 \mathrm{~cm}$ wide (Couceiro et al., 2006; Singh, 2005).

In the upper parts of $\mathrm{H}$. perforatum, several yellow flowers are produced, which are usually 1 to $2 \mathrm{~cm}$ wide. There are usually black dots on the edges of the petals. Blood red pigments are generated using crushed flowers. Flowers produced in late summer have capsules that contain tiny brown seeds (Crompton et al., 1988). Although this plant is native to Europe, it is distributed in all temperate regions in Asia, Australia, and North and South America.

In terms of medicinal and therapeutic properties, H. perforatum is the most important species of this genus, and today it is widely used in the treatment of mild-to moderate depression. The history of the traditional use of this medicinal plant dates back to over 2000 years ago. Antidepressant, anti-inflammatory, antimicrobial, antiviral, and anti-cancer properties have been reported for $H$. perforatum. Various studies have reported that $H$. perforatum has antiviral (Chen et al., 2019), wound healing (Samadi et al., 2010), antioxidant (Hakimouglu et al., 2007; Saddiqe et al., 2010) antimicrobial (Akhbari et al., 2012), and antibacterial properties (Tozlu et al., 2011).

Clinical trials have been conducted since the early 1990s on the effect of hypericin on the treatment of viral diseases, and experiments in this area show that the plant with low toxicity has significant effects against HIV-like viruses. So far, a wide range of biologically active compounds have been identified and reported in this plant, including hypericin and hyperforin.

Chemical research on the components of $H$. perforatum has led to the identification of several groups of pharmaceutically active compounds. The most important groups include naphthodianthrones (hypericin and pseudo-hypericin), phloroglucinols (hyperforin and adhyperforin), xanthones and flavonoids (such as phenylpropanes, flavanol glycosides and bioflavonoids) as well as essential oils. Various biological activities including wound healing, anti-anxiety and seizures, antiviral, antifungal, and antioxidant properties have been attributed to the compounds in the extract and essential oil of various species of the genus Hypericum (Bertoli et al., 2011). There are various reports on the antimicrobial activity of essential oils of different species of the genus Hypericum against bacteria and fungi of human and plant pathogens.

Selenium is one of the essential elements in the human diet. Although selenium has a variety of functions, its antioxidant and anti-cancer properties are of particular importance to humans. Selenium deficiency in the human diet also causes stunted growth, impaired bone metabolism, and thyroid dysfunction (Dumont et al., 2006). Selenium concentrations are usually higher in younger leaves than in older leaves during seedling growth (Galeas et al., 2007). Because selenium is so similar to sulfur, it competes with sulfur and enters plant cells through sulfate carriers in the root plasma membrane (Pilon-Smits et al., 2002).

Nanoparticles react with plants to cause a variety of physical and physiological changes, which significantly depend on the properties of the nanoparticles. The effectiveness of nanoparticles depends on their concentration and varies from plant to plant. The efficiency of nanoparticles depends on the chemical composition, size, surface area, reactivity, and concentration at which they respond positively. Nanoparticles have positive and negative effects on plant growth (Ekinci et al., 2014). Irmak (2017) conducted a study to investigate the effect of selenium application on plant growth and some quality parameters in peanut (Arachis hypogaea).

Hence, the main goal of this experiment was to evaluate the different selenium source application for with main focus morphopysiologial traits. Furthermore, we pointed to identify the appropriate concentration of Selenium that can develop essential oil and other features. 


\section{Methods}

This study was conducted in 2020 in Tehran, Iran. The study aimed to survey the effect of selenium and nano-selenium on vegetative growth and phytochemical and enzymatic properties of St. John's wort in the Tehran Municipality greenhouse. This study was conducted with three replications in a randomized complete block design. Each experimental unit consisted of 30 pots. Treatments included foliar application with different levels of selenium and nano-selenium. For this purpose, selenium at concentrations of 6, 8, 10 and $12 \mathrm{mg} / \mathrm{I}$ were foliar applied at the rosette stage. In addition, nano-selenium at concentrations of $6,8,10$, and $12 \mathrm{mg} / \mathrm{I}$ were foliar applied simultaneously with foliar application of sodium selenate. Foliar spraying of distilled water was used as a control (Fig. 1).

One-year-old seedlings of Topaz cultivar were obtained from Pakan Bazr Isfahan Company and planted in pots in March. After seedling establishment, foliar application was performed. Specimen was presented in central herbarium of Tehran University (Herbarium Code: TUH). (6398 No). Samples were authenticated by a botanist. Data collection and plant harvesting were performed at $50 \%$ flowering stage. Plant height, inflorescence length, number of inflorescences, inflorescence weight, shoot weight, root length, root weight, total biomass weight, essential oil percentage, hypericin and hyperforin content (Tawaha et al., 2010), selenium content (Singh et al., 1980), Relative Water Content (RWC), total chlorophyll, chlorophyll a and b, phenol content, DPPH, and proline content (Abbas et al., 2014), and the content of catalase (Velikova et al., 2000), peroxidase (Reuveni et al., 1992), and malondialdehyde (Dhindsa and Matowe, 1981), were measured and determined.

\section{Results}

The results of the analysis of variance showed that the effect of selenium and nano selenium fertilizers on plant height, inflorescence length, number of inflorescences, inflorescence weight, shoot weight, root length, total biomass weight, essential oil percentage, and hypericin and hyperforin content was significant at $1 \%$ level, and on the root weight at $5 \%$ level (Table 1 ).

Table 1

Effect of selenium and nano-selenium on morphophysiological characteristics and growth of St. John's wort using analysis of variance

\begin{tabular}{|c|c|c|c|c|c|c|c|c|c|c|c|}
\hline S.O.V & df & $\begin{array}{l}\text { plant } \\
\text { height }\end{array}$ & $\begin{array}{l}\text { inflorescence } \\
\text { length }\end{array}$ & $\begin{array}{l}\text { number of } \\
\text { inflorescences }\end{array}$ & $\begin{array}{l}\text { inflorescence } \\
\text { weight }\end{array}$ & $\begin{array}{l}\text { shoot } \\
\text { weight }\end{array}$ & $\begin{array}{l}\text { root } \\
\text { length }\end{array}$ & $\begin{array}{l}\text { root } \\
\text { weight }\end{array}$ & $\begin{array}{l}\text { total } \\
\text { biomass } \\
\text { weight }\end{array}$ & $\begin{array}{l}\text { essential } \\
\text { oil } \\
\text { percentage }\end{array}$ & $\begin{array}{l}\text { hypericin } \\
\text { content }\end{array}$ \\
\hline Replication & 2 & $75.69 *$ & $26.01 \mathrm{~ns}$ & $9 n s$ & $57.76^{* *}$ & $147.21^{\star *}$ & $87.84^{* \star}$ & $2.25 \mathrm{~ns}$ & $36.4^{\star}$ & $0.00 \mathrm{~ns}$ & $0.75^{\star \star}$ \\
\hline Treatment & 8 & $127.05^{\star \star}$ & $35.45^{\star \star}$ & $54 * *$ & 45.20 ** & 238.58 ** & $39.45^{\star \star}$ & $37.77^{*}$ & $773.08^{\star *}$ & $0.00 * \star$ & $3.53^{\star *}$ \\
\hline Error & 16 & 7.22 & 8.52 & 5.25 & 5.58 & 2.69 & 0.62 & 5.46 & 10.59 & 0.00 & 0.06 \\
\hline \%CV. & & 5.52 & 13.59 & 22.91 & 5.35 & 2.90 & 3.4 & 13.03 & 3.66 & 23.06 & 10.82 \\
\hline
\end{tabular}

Ns, * and ** indicate non-significance and significance at the level of 5 and $1 \%$, respectively.

Continuous of Table (1).

\begin{tabular}{|c|c|c|c|c|c|c|c|c|c|c|c|c|}
\hline S.o.V & df & selenium & RWC & $\begin{array}{l}\text { chlorophylls } \\
\text { a }\end{array}$ & $\begin{array}{l}\text { chlorophylls } \\
\text { b }\end{array}$ & $\begin{array}{l}\text { chlorophylls } \\
\text { total }\end{array}$ & phenol & proline & CAT & POD & MDA & DPPH \\
\hline Replication & 2 & $378.56^{* *}$ & $272.03^{\star *}$ & $0.19 * \star$ & $0.006^{* \star}$ & $0.26^{\star \star}$ & $2.08^{\star \star}$ & $44.44^{\star \star}$ & $0.002^{\star \star}$ & $0.40 *$ & $3321.6 *$ & $54.76^{\star}$ \\
\hline Treatment & 8 & $16804.1^{\star \star \star}$ & $508.77 * \star$ & $0.09 * \star$ & $0.02^{\star \star}$ & $0.17 \star \star$ & $3.07 \star \star$ & $614.33^{* *}$ & $0.004^{\star \star}$ & $0.61^{\star \star}$ & $201147^{\star \star}$ & $350.0 \varepsilon$ \\
\hline Error & 16 & 21.81 & 0.39 & 0.00 & 0.00 & 0.00 & 0.29 & 0.98 & 0.00 & 0.07 & 769.53 & 4.59 \\
\hline$\% C V$. & & 3.84 & 3.88 & 4.00 & 7.04 & 3.50 & 13.06 & 3.62 & 13.48 & 15.14 & 9.28 & 3.83 \\
\hline
\end{tabular}

RWC: Relative Water Content, CTA: Catalas, POD: Proxidase, MDA: Malondialdehyde

$\mathrm{Ns}$, * and ** indicate non-significance and significance at the level of 5 and $1 \%$, respectively.

The effect of selenium and nano-selenium on the amount of selenium accumulation in the plant, relative leaf water content, the content of chlorophylls a, b, and total, the phenol and proline content, and production and accumulation of catalase, peroxidase, malondialdehyde, and DPPH enzymes were statistically significant at the $1 \%$ level (Table 1$)$.

Comparison of means showed that the highest plant height was obtained from control treatment $(58 \mathrm{~cm})$ and foliar application of $6 \mathrm{mg} / \mathrm{l}$ selenium (56.4 $\mathrm{cm})$ (Table 2). Inflorescence length was the highest in control treatment and two treatment levels of 6 and $8 \mathrm{mg} / \mathrm{l} \mathrm{selenium}$ and nano-selenium (Table 2). The number of inflorescences was the highest in the control treatment and the first treatment level (6 mg/l) of selenium and nano-selenium (Table 2). 
Table 2

Comparison of means of the effect of selenium and nano-selenium on morphophysiological characteristics and growth of St. John's wort

\begin{tabular}{|c|c|c|c|c|c|c|c|c|c|c|c|}
\hline & $\begin{array}{l}\text { plant } \\
\text { height }\end{array}$ & $\begin{array}{l}\text { inflorescence } \\
\text { length }\end{array}$ & $\begin{array}{l}\text { number of } \\
\text { inflorescences }\end{array}$ & $\begin{array}{l}\text { inflorescence } \\
\text { weight }\end{array}$ & $\begin{array}{l}\text { shoot } \\
\text { weight }\end{array}$ & $\begin{array}{l}\text { root } \\
\text { length }\end{array}$ & $\begin{array}{l}\text { root } \\
\text { weight }\end{array}$ & $\begin{array}{l}\text { total } \\
\text { biomass } \\
\text { weight }\end{array}$ & $\begin{array}{l}\text { essential } \\
\text { oil } \\
\text { percentage }\end{array}$ & $\begin{array}{l}\text { hypericin } \\
\text { content }\end{array}$ & $\begin{array}{l}\text { hyperforin } \\
\text { content }\end{array}$ \\
\hline & $\mathrm{cm}$ & $\mathrm{cm}$ & & $\mathrm{g} /$ plant & $\mathrm{g} /$ plant & $\mathrm{cm}$ & $\mathrm{g} /$ plant & $\mathrm{g} /$ plant & $\%$ & $\begin{array}{l}\mathrm{mg} / \mathrm{g} \\
\mathrm{dry} \\
\text { matter }\end{array}$ & $\begin{array}{l}\mathrm{mg} / \mathrm{g} \text { dry } \\
\text { matter }\end{array}$ \\
\hline co & $58 a$ & $25.5 a$ & $16 a$ & $21 a$ & $70 a$ & $27 a$ & $24 a$ & $115 a$ & $0.14 a$ & $2.7 \mathrm{~cd}$ & $44 c$ \\
\hline si6 & $56.4 a$ & $25 a b$ & $14 a b$ & $17 b$ & $66 \mathrm{~b}$ & $26 a b$ & $22 a b$ & $105 b$ & $0.1 \mathrm{~b}$ & $3.2 b$ & $50 \mathrm{~b}$ \\
\hline 8 & $50 \mathrm{bc}$ & $24 a b$ & $10 \mathrm{~cd}$ & $15 c$ & $60 c$ & $25 b c$ & $19 b c$ & $94 \mathrm{~cd}$ & $0.09 b c$ & $3.8 a$ & $44 c$ \\
\hline 10 & $48.5 b c$ & $20 \mathrm{bcd}$ & $8 \mathrm{de}$ & $12 d$ & $55 d$ & $22 d$ & $17.5 c$ & $84.5 \mathrm{e}$ & $0.07 \mathrm{bcde}$ & $1.5 \mathrm{e}$ & $57 a$ \\
\hline 12 & $41 \mathrm{de}$ & $18 \mathrm{~cd}$ & $6 \mathrm{ef}$ & $10 e$ & $47 e$ & $20 \mathrm{ef}$ & $16.5 c$ & $73.5 f$ & $0.05 \mathrm{de}$ & $1 f$ & $33 e$ \\
\hline nanosi6 & $51.5 b$ & $23.8 a b$ & $14 a b$ & $17.5 b$ & $62 c$ & $25 b c$ & $18 b c$ & $97.5 c$ & $0.1 b$ & $3 b c$ & $46 c$ \\
\hline 8 & $49 b$ & $22.5 \mathrm{abc}$ & $12 \mathrm{bc}$ & $15 c$ & $57 d$ & $23.8 \mathrm{c}$ & $17.5 \mathrm{c}$ & $89.5 \mathrm{de}$ & $0.08 \mathrm{bcd}$ & $2.5 \mathrm{~d}$ & $41 d$ \\
\hline 10 & $45.6 \mathrm{~cd}$ & $18.5 \mathrm{~cd}$ & $6 e f$ & $12 d$ & $48 e$ & $21 \mathrm{de}$ & $15 \mathrm{~cd}$ & $75 f$ & $0.06 \mathrm{cde}$ & 1.2ef & $34 \mathrm{e}$ \\
\hline 12 & $38 e$ & $16 d$ & $4 f$ & $9 e$ & $44 f$ & $18.8 f$ & $12 d$ & $65 \mathrm{~g}$ & $0.04 \mathrm{e}$ & $0.8 f$ & $26 f$ \\
\hline
\end{tabular}

Continuous of Table (2).

\begin{tabular}{|c|c|c|c|c|c|c|c|c|c|c|c|}
\hline & selenium & RWC & $\begin{array}{l}\text { chlorophylls } \\
\text { a }\end{array}$ & $\begin{array}{l}\text { chlorophylls } \\
\text { b }\end{array}$ & $\begin{array}{l}\text { chlorophylls } \\
\text { total }\end{array}$ & phenol & proline & CAT & POD & MDA & DPPH \\
\hline & $\mu \mathrm{g} / \mathrm{g} \mathrm{dw}$ & $\%$ & $\mathrm{~g} / 100 \mathrm{~g} \mathrm{FM}$ & g/100 g FM & g/100 g FM & $\begin{array}{l}\mathrm{Mg} / \mathrm{I} \mathrm{FW} \\
\text { extract }\end{array}$ & $\mathrm{FW}_{\mathrm{FW}} \mathrm{mol} / \mathrm{g}$ & $\begin{array}{l}\mathrm{u} / \mathrm{g} \\
\mathrm{FW} / \mathrm{min}\end{array}$ & $\begin{array}{l}\mathrm{U} / \mathrm{g} \\
\mathrm{FW} / \mathrm{min}\end{array}$ & $\mathrm{FW}_{\mathrm{FW}}^{\mathrm{mM}}$ & $\%$ \\
\hline $\mathrm{co}$ & $37 \mathrm{~h}$ & $84 a$ & $0.77 e$ & $0.26 \mathrm{~d}$ & $1.03 e$ & $3.5 \mathrm{c}$ & $12 \mathrm{~h}$ & $0.05 \mathrm{e}$ & $1.1 \mathrm{~d}$ & $68 d$ & $35 f$ \\
\hline si6 & $49.5 \mathrm{~g}$ & $76 b$ & $0.96 \mathrm{~d}$ & $0.3 \mathrm{~cd}$ & $1.26 \mathrm{~d}$ & $3.23 c$ & $15 g$ & $0.07 \mathrm{de}$ & $1.3 d$ & $92 d$ & $47 e$ \\
\hline 8 & $79 f$ & $75 b$ & $1.02 \mathrm{~d}$ & $0.32 b c$ & $1.34 \mathrm{~d}$ & $3.31 c$ & $25 d$ & $0.07 \mathrm{de}$ & $2 a b$ & $103 d$ & $56 c$ \\
\hline 10 & $120 d$ & $70 c$ & $1.13 c$ & $0.35 b$ & $1.48 c$ & $3.28 c$ & $33 c$ & $0.1 \mathrm{bc}$ & $2.3 a$ & $185 c$ & $68 a$ \\
\hline 12 & 137c & $61 f$ & $1.23 \mathrm{~b}$ & $0.28 \mathrm{~cd}$ & $1.51 \mathrm{c}$ & $4.54 b$ & $48 b$ & $0.15 a$ & $1.5 \mathrm{~cd}$ & $590 \mathrm{~b}$ & $61 b$ \\
\hline nanosi6 & $94 \mathrm{e}$ & $75.5 b$ & $1 d$ & $0.32 \mathrm{bc}$ & $1.32 \mathrm{~d}$ & $3.41 \mathrm{c}$ & $17 f$ & $0.08 \mathrm{~cd}$ & $1.4 \mathrm{~cd}$ & $110 d$ & $52 d$ \\
\hline 8 & $122.63 d$ & $68.4 \mathrm{~d}$ & $1.19 b c$ & $0.36 b$ & $1.55 c$ & $4.66 \mathrm{~b}$ & $19 e$ & $0.09 \mathrm{~cd}$ & $1.8 \mathrm{bc}$ & $222 c$ & $71 a$ \\
\hline 10 & $167.52 b$ & $66 \mathrm{e}$ & $1.25 \mathrm{ab}$ & $0.46 a$ & $1.71 b$ & $5.2 a b$ & $26 d$ & $0.12 b$ & $2.4 a$ & $695 a$ & $57 c$ \\
\hline 12 & $287.52 a$ & $58 \mathrm{~g}$ & $1.32 a$ & $0.49 a$ & $1.81 \mathrm{a}$ & $6 a$ & $52 a$ & $0.16 a$ & $1.5 \mathrm{~cd}$ & $624 b$ & $55 \mathrm{~cd}$ \\
\hline \multicolumn{12}{|c|}{ RWC: Relative Water Content, CTA: Catalas, POD: Proxidase, MDA: Malondialdehyde } \\
\hline
\end{tabular}

The highest inflorescence weight (21 g/plant), shoot weight (70 g/plant), root weight ( $27 \mathrm{~g} / \mathrm{plant})$, total biomass weight (115 g/plant), and essential oil content $(0.14 \%)$ was obtained from the control treatment. The highest root weight was obtained from the control treatment $(24 \mathrm{~cm})$ and foliar application of 6 $\mathrm{mg} / \mathrm{l}$ selenium $(22 \mathrm{~cm})$. The highest accumulation of hypericin $(3.8 \mathrm{mg} / \mathrm{g}$ dry matter) was obtained from the foliar application of $8 \mathrm{mg} / \mathrm{l}$ selenium. The highest accumulation of hyperforin ( $57 \mathrm{mg} / \mathrm{g}$ dry matter) was obtained from the foliar application of $10 \mathrm{mg} / \mathrm{l}$ selenium.

It was observed that the highest accumulation of selenium $(287.52 \mu \mathrm{g} / \mathrm{g}$ dry matter), the highest content of chlorophyll a ( $1.32 \mathrm{~g})$, chlorophyll b ( $0.49 \mathrm{~g})$ total chlorophyll $(1.81 \mathrm{~g})$, phenol $(6 \mathrm{mg})$, and proline $(52 \mu \mathrm{mol} / \mathrm{g})$ were obtained in the foliar application of $12 \mathrm{mg} / \mathrm{l}$ nano-selenium. The highest catalase content was obtained from the foliar application of $12 \mathrm{mg} / \mathrm{I}$ nano-selenium $(0.16 \mathrm{u} / \mathrm{g} \mathrm{FW} / \mathrm{min})$ and foliar application of $12 \mathrm{mg} / \mathrm{l} \mathrm{selenium} \mathrm{(0.15} \mathrm{u/g} \mathrm{FW/min).}$

Peroxidase was higher in the foliar application of 8 and $10 \mathrm{mg} / \mathrm{l}$ selenium and $10 \mathrm{mg} / \mathrm{l}$ nano-selenium than other treatments. The highest malondialdehyde leakage ( $695 \mathrm{u} / \mathrm{g} \mathrm{FW} / \mathrm{min}$ ) was obtained from the $10 \mathrm{mg} / \mathrm{l}$ nano-selenium treatment. The highest levels of antioxidant enzyme (71 and $68 \%$ ) were obtained from the foliar application of $8 \mathrm{mg} / \mathrm{l}$ selenium and $10 \mathrm{mg} / \mathrm{l}$ nano-selenium, respectively.

The study of simple relationships between traits showed a significant positive correlation between the plant height and inflorescence length, number of inflorescences, inflorescence weight, shoot weight, root length, root weight, total biomass weight, essential oil percentage, the content of hypericin and hyperforin, relative water content, and peroxidase at the level of $1 \%$. The plant height was also significantly correlated with the amount of selenium, chlorophyll a, b, and total chlorophyll, phenol, proline, catalase, malondialdehyde, and DPPH (Table 3). Similar to the plant height, the inflorescence length, 
number of inflorescences, inflorescence weight, shoot weight, root length, root weight, essential oil percentage, and amount of hypericin and hyperforin had a significant correlation with other traits (Table 3).

Table 3

Simple correlation of the effects of selenium and nano-selenium on morphophysiological characteristics and growth of St. John's wort

\begin{tabular}{|c|c|c|c|c|c|c|c|c|c|c|c|}
\hline & $\begin{array}{l}\text { plant } \\
\text { height }\end{array}$ & $\begin{array}{l}\text { inflorescence } \\
\text { length }\end{array}$ & $\begin{array}{l}\text { number of } \\
\text { inflorescences }\end{array}$ & $\begin{array}{l}\text { inflorescence } \\
\text { weight }\end{array}$ & $\begin{array}{l}\text { shoot } \\
\text { weight }\end{array}$ & $\begin{array}{l}\text { root } \\
\text { length }\end{array}$ & $\begin{array}{l}\text { root } \\
\text { weight }\end{array}$ & $\begin{array}{l}\text { total } \\
\text { biomass } \\
\text { weight }\end{array}$ & $\begin{array}{l}\text { essential oil } \\
\text { percentage }\end{array}$ & $\begin{array}{l}\text { hypericin } \\
\text { content }\end{array}$ & $\begin{array}{l}\text { hyperforin } \\
\text { content }\end{array}$ \\
\hline & 1 & 2 & 3 & 4 & 5 & 6 & 7 & 8 & 9 & 10 & 11 \\
\hline 1 & 1.00 & & & & & & & & & & \\
\hline 2 & $0.83^{\star \star}$ & 1.00 & & & & & & & & & \\
\hline 3 & $0.84^{\star \star}$ & $0.77 * \star$ & 1.00 & & & & & & & & \\
\hline 4 & $0.55^{\star \star}$ & $0.45^{\star}$ & $0.64^{\star \star}$ & 1.00 & & & & & & & \\
\hline 5 & $0.91^{\star \star}$ & $0.85^{\star \star}$ & $0.87^{\star \star \star}$ & $0.57^{\star \star *}$ & 1.00 & & & & & & \\
\hline 6 & $0.90^{\star \star \star}$ & $0.81^{\star \star}$ & $0.79 * \star$ & $0.35 \mathrm{~ns}$ & $0.93^{\star \star}$ & 1.00 & & & & & \\
\hline 7 & $0.71^{\star \star}$ & $0.57^{\star \star *}$ & $0.71^{\star \star}$ & $0.61^{\star \star}$ & $0.82^{\star \star}$ & $0.68^{\star *}$ & 1.00 & & & & \\
\hline 8 & $0.86^{\star *}$ & $0.77^{\star \star}$ & $0.87^{\star \star}$ & $0.76^{\star \star}$ & $0.95^{\star \star}$ & $0.81^{\star \star}$ & $0.90^{* \star}$ & 1.00 & & & \\
\hline 9 & $0.80^{\star \star}$ & $0.81^{\star \star}$ & $0.79 \star \star$ & $0.62^{\star \star}$ & $0.89 * *$ & $0.78^{\star \star}$ & $0.76^{\star \star}$ & $0.89^{\star \star *}$ & 1 & & \\
\hline 10 & $0.77^{\star \star *}$ & 0.82 & $0.75^{\star \star}$ & $0.52^{\star \star}$ & $0.83^{\star \star}$ & $0.82^{\star \star}$ & $0.62^{\star \star}$ & $0.79 * \star$ & $0.73^{\star \star}$ & 1.00 & \\
\hline 11 & $0.71^{\star \star}$ & $0.59 * *$ & $0.54 * \star$ & $0.31 \mathrm{~ns}$ & $0.70^{* \star}$ & $0.63^{* \star}$ & $0.56^{\star \star}$ & $0.64^{\star \star}$ & $0.56^{\star \star}$ & $0.58^{* *}$ & 1.00 \\
\hline 12 & -0.78 ** & $-0.68^{\star \star}$ & $-0.75^{\star \star}$ & $-0.74^{\star *}$ & $-0.79 \star \star$ & $-0.66^{\star \star}$ & $-0.81^{\star \star}$ & $-0.87^{\star \star}$ & $-0.74^{\star \star}$ & $-0.73^{\star \star}$ & $-0.68^{\star *}$ \\
\hline 13 & $0.91^{\star \star}$ & $0.78^{\star \star}$ & $0.82^{\star \star}$ & $0.60 \star \star$ & $0.96^{\star \star}$ & $0.90 * \star$ & $0.79 * \star$ & $0.93^{\star \star}$ & $0.86^{\star \star}$ & $0.79 * \star$ & $0.70^{\star \star \star}$ \\
\hline 14 & $-0.47^{\star}$ & $-0.36 n s$ & $-0.50 *$ & $-0.94^{\star \star}$ & $-0.49 *$ & $-0.25 \mathrm{~ns}$ & $-0.60 * \star$ & -0.70 ** & $-0.59 * \star$ & $-0.44^{\star}$ & $-0.32 n s$ \\
\hline 15 & $-0.48^{\star}$ & $-0.39 *$ & $-0.55^{\star \star}$ & $-0.65^{\star \star}$ & $-0.50 *$ & $-0.34 \mathrm{~ns}$ & $-0.62^{\star \star}$ & $-0.63^{* *}$ & $-0.46^{\star}$ & $-0.44^{\star}$ & $-0.43^{\star}$ \\
\hline 16 & $-0.50 *$ & $-0.39 *$ & $-0.54^{\star \star}$ & $-0.91^{\star \star}$ & $-0.53^{\star \star}$ & $-0.29 n s$ & $-0.64^{\star \star}$ & $-0.72^{\star \star}$ & $-0.59 * \star$ & $-0.47^{\star}$ & $-0.37^{\star}$ \\
\hline 17 & $-0.56^{\star \star}$ & $-0.53^{\star \star}$ & $-0.53^{\star \star}$ & $-0.67 * \star$ & $-0.53^{\star *}$ & $-0.34 \mathrm{~ns}$ & $-0.59 * \star$ & $-0.64^{\star *}$ & $-0.56 * \star$ & $-0.58^{\star \star}$ & $-0.66^{* *}$ \\
\hline 18 & $-0.78 \star \star$ & $-0.67 \star \star$ & $-0.78 \star \star$ & $-0.84^{\star \star}$ & $-0.76^{\star \star}$ & $-0.64^{\star \star}$ & $-0.66^{\star \star}$ & $-0.84^{\star \star}$ & $-0.75^{\star \star}$ & $-0.72^{\star \star}$ & $-0.54^{\star}$ \\
\hline 19 & $-0.71 \star \star$ & $-0.54^{\star \star}$ & $-0.71^{\star *}$ & $-0.87^{\star *}$ & $-0.68^{\star \star}$ & $-0.52^{\star}$ & $-0.67^{\star \star}$ & $-0.81^{\star *}$ & $-0.72^{\star \star}$ & $-0.71 \star \star$ & $-0.56^{\star}$ \\
\hline 20 & $-0.17 n s$ & $-0.24 n s$ & $-0.43^{\star}$ & $-0.51^{*}$ & $-0.24 n s$ & $-0.06 n s$ & $-0.19 n s$ & $-0.33 n s$ & $-0.35 n s$ & $-0.15 n s$ & $0.16 \mathrm{~ns}$ \\
\hline 21 & $-0.73^{\star \star}$ & $-0.72^{\star \star}$ & $-0.76^{\star \star}$ & $-0.72^{\star \star}$ & $-0.82^{\star \star}$ & $-0.67^{\star \star}$ & $-0.69 * *$ & $-0.86^{\star *}$ & $-0.73^{\star \star}$ & $-0.81^{\star *}$ & $-0.76^{\star \star}$ \\
\hline 22 & $-0.39 *$ & $-0.33 \mathrm{~ns}$ & $-0.42^{\star}$ & $-0.63^{\star *}$ & $-0.45^{\star}$ & $-0.28 n s$ & $-0.48^{\star}$ & $-0.56^{\star}$ & $-0.56^{\star}$ & $-0.28 n s$ & $0.04 \mathrm{~ns}$ \\
\hline
\end{tabular}

Continuous of Table (3). 


\begin{tabular}{|c|c|c|c|c|c|c|c|c|c|c|c|}
\hline & selenium & RWC & chlorophylls a & chlorophylls b & chlorophylls total & phenol & proline & CAT & POD & MDA & DPPH \\
\hline & 12 & 13 & 14 & 15 & 16 & 17 & 18 & 19 & 20 & 21 & 22 \\
\hline 12 & 1.00 & & & & & & & & & & \\
\hline 13 & $-0.80 * *$ & 1.00 & & & & & & & & & \\
\hline 14 & $0.73^{\star *}$ & $-0.53^{* *}$ & 1 & & & & & & & & \\
\hline 15 & $0.83^{* \star}$ & $-0.49 *$ & $0.71^{\star *}$ & 1 & & & & & & & \\
\hline 16 & $0.8^{\star \star}$ & $-0.55^{\star}$ & $0.98^{* *}$ & $0.84^{\star *}$ & 1 & & & & & & \\
\hline 17 & $0.8^{* \star}$ & $-0.52^{*}$ & $0.78^{\star *}$ & $0.73^{\star *}$ & $0.81^{* \star}$ & 1 & & & & & \\
\hline 18 & $0.81^{* *}$ & $-0.77^{\star *}$ & $0.69 * *$ & $0.46^{*}$ & $0.66^{* *}$ & $0.59 * *$ & 1 & & & & \\
\hline 19 & $0.83^{* *}$ & $-0.71^{\star *}$ & $0.84^{\star *}$ & $0.63^{\star *}$ & $0.83^{\star *}$ & $0.78^{\star \star}$ & $0.87^{\star *}$ & 1 & & & \\
\hline 20 & $0.26 \mathrm{~ns}$ & $-0.15 n s$ & $0.52^{*}$ & $0.51^{*}$ & $0.55^{\star \star}$ & $0.25 \mathrm{~ns}$ & $0.19 \mathrm{~ns}$ & $0.28 \mathrm{~ns}$ & 1 & & \\
\hline 21 & $0.8^{* *}$ & -0.78 ** & $0.7 * \star$ & $0.64^{*}$ & $0.72^{\star *}$ & $0.77^{\star *}$ & $0.71^{\star \star}$ & $0.82^{\star \star}$ & $0.29 \mathrm{~ns}$ & 1 & \\
\hline 22 & $0.38 *$ & $-0.49 *$ & $0.68^{*}$ & $0.33 \mathrm{~ns}$ & $0.61^{\star *}$ & $0.31 \mathrm{~ns}$ & 0.41 * & $0.44^{*}$ & $060 \star \star$ & $0.29 \mathrm{~ns}$ & 1 \\
\hline \multicolumn{12}{|c|}{ RWC: Relative Water Content, CTA: Catalas, POD: Proxidase, MDA: Malondialdehyde } \\
\hline $\mathrm{N}$ & $d * *$ inc & non & 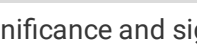 & can & of $5 \mathrm{a}$ & tively. & & & & & \\
\hline
\end{tabular}

It was observed that the content of selenium had a significant negative correlation with morphological traits and yield of different organs and a significant positive correlation with the number of enzymes, chlorophylls, phenol, and proline. In contrast to selenium, relative water content had a significant positive correlation with agronomic traits and yields and a significant negative correlation with the number of enzymes, chlorophylls, phenol, and proline.

As can be seen in Table 3, physiological traits such as chlorophylls, enzymes, phenol, and proline had a significant positive correlation with each other.

\section{Discussion}

Considering climate change (Peteet, 2000; Prentice et al., 1991), greenhouse gases phenomenon (Avand et al., 2020; Griggs \& Noguer, 2002), and drought (Aimar et al., 2014; Aranjuelo et al., 2011; Naumburg et al., 2004) in the regions of the world and especially in Iran, introducing new crops with economic value and high water use efficiencies such as $H$. perforatum and the study of its response to different treatment levels is one of the important research priorities in Iran and the world. The significant difference between replications was because of uneven greenhouse conditions, showing that the random complete blocks design was correctly selected. Moreover, the significant differences between traits (Table 1) show the selection of the hypothesis, the subject of research, and treatments, and the importance of research on $H$. perforatum and the introduced treatments.

Comparison of means showed that the highest plant height was obtained from control treatment and foliar application of $6 \mathrm{mg} / \mathrm{l}$ selenium (Table 2), and the plant height decreased with increasing the concentration of treatments. The decreased height with selenium treatments indicates the intolerance of $H$. perforatum to selenium stress. Because the number of inflorescences is affected by environmental and genetic factors and tillers, so by applying environmental treatments, the role of each of the environmental and genetic factors can be revealed. In this study, it was observed that inflorescences are more affected by environmental factors. In this study, the results of the effect of selenium as an environmental factor on the number of tillers are shown in Table 2. These results are consistent with the findings Soleimani et al., (2017) and Uddin et al., (2016) on the effect of environmental and genetic factors on the number of tillers. The highest shoot weight, root weight, and total biomass weight were obtained from the control treatment. Therefore, the results of this part of the research show that $H$. perforatum is intolerant to selenium and nano-selenium.

The studied plant appears to cope with selenium and nano-selenium stress with changes in morphological traits (Thomas and Gausling, 2000), decrease in photosynthetic and transpiration organs, increase in regulatory osmolytes (Kivimäenpää et al., 2003), changes in the synthesis of materials within the plant (Flexas et al., 2009), increased water-absorbing organs (Dambolena et al., 2010) and finally allocation of photosynthetic material for active water absorption by energy (Slattery and Ort, 2015), all of which will reduce plant shoot yield as occurred for $H$. perforatum. The highest essential oil percentage was obtained from the control treatment, while the content of hypericin and hyperforin increased at low levels of selenium and nano-sellenium.

Basically, in plants, when they are stressed, the pentose phosphate pathway becomes more active and the production of essential oil increases. It has also been reported that plants under conditions of severe stress increase the percentage of other regulating compounds and osmolytes such as proline instead of essential oils and this is more evident in sensitive plants. The highest accumulation of hypericin and hyperforin was obtained from the foliar application of 8 $\mathrm{mg} / \mathrm{l}$ and $10 \mathrm{mg} / \mathrm{l}$ selenium, respectively, showing that the use of small amounts of these two elements can be useful for producing high-quality plants. Catalase enzyme was highest in the foliar application of $12 \mathrm{mg} / \mathrm{l}$ nano selenium and the foliar application of $12 \mathrm{mg} / \mathrm{l}$ selenium, which indicates the production of this enzyme at high levels of selenium and nano selenium. However, peroxidase production is faster than catalase in the plant, and it seems that at the same time as peroxidase production, malondialdehyde leakage reaches its maximum.

The results showed that in the foliar application of $12 \mathrm{mg} / \mathrm{l}$ nano-selenium, the maximum accumulation of selenium and the highest amount of chlorophyll a, chlorophyll b, total chlorophyll, phenol, and proline were obtained. Therefore, St. John's wort was a plant that absorbs selenium and nano-selenium, and despite the increase in chlorophyll content, a decrease in yield and an increase in antioxidant activity were observed. In general, the effect of selenium and 
nano-selenium particles depends on the composition, size, surface area, reactivity, and concentration in which they respond positively and have positive and negative effects on plant growth and development (Ekinci et al., 2014). In our study, the same positive and negative results were observed on different traits, and in this regard, the results are consistent with the research of Ekinci et al, (2014). The results of this study are similar to the research on Lemongrass (Melissa officinalis L.) in terms of decreasing yield and increasing the concentration of chlorophyll a and b and the activity of catalase and malondialdehyde in the $10 \mathrm{mg} / \mathrm{l}$ treatment.

Simple relationships between traits showed a significant positive correlation between morphological traits and yields.

\section{Conclusion}

Therefore, if the purpose of producing $H$. perforatum is to use the shoots and essential oils of the plant, then the use of selenium and nano-selenium is not recommended at all and should not be used. If the goal is to produce high hypericin, high hyperforin, and also the accumulation of selenium in the plant, the use of treatments of 6 and $8 \mathrm{mg} / \mathrm{l}$ of selenium and nano-selenium could be used. A significant negative correlation between selenium content with morphological traits and yield of different organs and a positive correlation with the content of enzymes, chlorophylls, phenol, and proline shows that selenium causes stress in the plant and therefore plant growth is reduced and instead increases the production of antioxidant enzymes and osmolytes. There is a significant negative correlation between selenium content with morphological traits and function of different organs and a positive correlation with the number of enzymes, chlorophylls, phenol and proline indicates that selenium causes stress in the plant and therefore plant growth is reduced and instead production increases antioxidant enzymes and osmolytes.

\section{Abbreviations}

S: Selenium, RWC: Relative Water Content, CTA: Catalas, POD: Proxidase, MDA: Malondialdehyde

\section{Declarations}

\section{Ethics approval and consent to participate: Not applicable}

\section{Consent for publication: Not applicable.}

Availability of data and materials: Not available

Competing interests: The authors declare no conflict of interest.

Funding: This study was not financially supported by organization or ect.

Author contributions: M.R.N. and V.A.; Conceptualization, Methodology, Formal analysis and investigation, M.R.N. Writing-original draft preparation, M.R.N., V.A., F.Z.H. and K.L.; Writing - review and editing, V.A., F.Z.H. and K.L.; Resources, Supervision; All authors have read and approved the manuscript

Acknowledgements: Not applicable.

\section{References}

1. Abbas SR, Ahmad SD, Sabir SM, Shah AH. Detection of drought tolerant sugarcane genotypes (Saccharum officinarum) using lipid peroxidation, antioxidant activity, glycine-betaine and proline contents. J soil Sci plant Nutr. 2014;14:233-43. http://dx.doi.org/10.4067/S0718-95162014005000019.

2. Aimar D, Calafat M, Andrade AM, Carassay L, Bouteau F, Abdala G, Molas ML. Drought effects on the early development stages of Panicum virgatum L.: cultivar differences. Biomass Bioenerg. 2014;66:49-59. https://doi.org/10.1016/j.biombioe.2014.03.004.

3. Akhbari M, Batooli H, Mozdianfard M. Comparative study of composition and biological activities of SDE prepared essential oils from flowers and fruits of two Hypericum species from central Iran. Nat Prod Res. 2012;26:193-202. https://doi.org/10.1080/14786419.2010.534994.

4. Aranjuelo I, Molero G, Erice G, Avice JC, Nogués S. Plant physiology and proteomics reveals the leaf response to drought in alfalfa (Medicago sativa L.). J Exp Bot. 2011;62:111-23. https://doi.org/10.1093/jxb/erq249.

5. Avand M, Moradi H, Ramazanzadeh M. (2020). Using machine learning models, remote sensing, and GIS to investigate the effects of changing climates and land uses on flood probability. J of Hydrol 125663. https://doi.org/10.1016/j.jhydrol.2020.125663.

6. Bertoli A, Cirak C, Silva JAT. Hypericum species as sources of valuable essential oils. Med. Aromat. Plant Sci Biotechnol. 2011;5:29-47.

7. Chen H, Muhammad I, Zhang Y, Ren Y, Zhang R, Huang X, Diao L, Liu H, Li X, Sun X, others, (2019). Antiviral Activity Against Infectious Bronchitis Virus and Bioactive Components of Hypericum perforatum L. Front. Pharmacol. 10, 1272. https://doi.org/10.3389/fphar.2019.01272.

8. Couceiro MA, Afreen F, Zobayed SMA, Kozai T. Variation in concentrations of major bioactive compounds of St. John's wort: Effects of harvesting time, temperature and germplasm. Plant Sci. 2006;170:128-34. https://doi.org/10.1016/j.plantsci.2005.08.011.

9. Crockett SL. (2010). Essential oil and volatile components of the genus Hypericum (Hypericaceae). Nat Prod Commun 5. https://doi.org/10.1177/1934578X1000500926. 
10. Crompton CW, Hall IV, Jensen KIN, Hildebrand PD. The biology of Canadian weeds. 83. Hypericum perforatum L. Can J Plant Sci. 1988;68:149-62. https://doi.org/10.4141/cjps88-015.

11. Dambolena JS, Zunino MP, Lucini El, Olmedo R, Banchio E, Bima PJ, Zygadlo JA. Total phenolic content, radical scavenging properties, and essential oil composition of Origanum species from different populations. J Agric Food Chem. 2010;58:1115-20. https://doi.org/10.1021/jf903203n.

12. Dhindsa RS, Matowe W. Drought tolerance in two mosses: correlated with enzymatic defence against lipid peroxidation. J Exp Bot. 1981;32:79-91. https://doi.org/10.1093/jxb/32.1.79.

13. Dumont E, Vanhaecke F, Cornelis R. (2006). Selenium speciation from food source to metabolites: a critical review. Anal Bioanal Chem $385,1304-23$. https:// 10.1007/s00216-006-0529-8.

14. Ekinci M, Dursun A, Yildirim E, Parlakova F. Effects of nanotechnology liquid fertilizers on the plant growth and yield of cucumber (Cucumis sativus L.). Acta Sci Pol Hortorum Cultus. 2014;13:135-41.

15. Flexas J, Barón M, Bota J, Ducruet JM, Gallé A, Galmés J, Jiménez M, Pou A, Ribas-Carbó M, Sajnani C, others, (2009). Photosynthesis limitations during water stress acclimation and recovery in the drought-adapted Vitis hybrid Richter-110 (V. berlandieri\$times\$ V. rupestris). J. Exp. Bot. 60, $2361-2377$. https://doi.org/10.1093/jxb/erp069 .

16. Galeas ML, Zhang LH, Freeman JL, Wegner M, Pilon-Smits EAH. Seasonal fluctuations of selenium and sulfur accumulation in selenium hyperaccumulators and related nonaccumulators. New Phytol. 2007;173:517-25. https://doi.org/10.1111/j.1469-8137.2006.01943.x.

17. Griggs DJ, Noguer M. Climate change (2001): the scientific basis. Contribution of working group I to the third assessment report of the intergovernmental panel on climate change. Weather. 2002;57:267-9. https://doi.org/10.1256/004316502320517344.

18. Hakimouglu F, Kizil G, Kanay Z, Kizil M, Isi H. The effect of ethanol extract of Hypericum lysimachioides on lipid profile in hypercholesterolemic rabbits and its in vitro antioxidant activity. Atherosclerosis. 2007;192:113-22. https://doi.org/10.1016/j.atherosclerosis.2006.07.013.

19. Irmak S. Effects of selenium application on plant growth and some quality parameters in peanut (Arachis hypogaea). Pakistan J Biol Sci. 2017;20:92-9. https://doi.org/10.3923/pjbs.2017.92.99.

20. Kivimäenpää M, Sutinen S, Karlsson PE, Selldén G. Cell structural changes in the needles of Norway spruce exposed to long-term ozone and drought. Ann Bot. 2003;92:779-93. https://doi.org/10.1093/aob/mcg202.

21. Mozaffarian V. A dictionary of Iranian plant names. Latin, English, Persian. Farhang Mo'aser; 1996.

22. Naumburg E, Loik ME, Smith SD. Photosynthetic responses of Larrea tridentata to seasonal temperature extremes under elevated CO2. New Phytol. 2004;162:323-30. https://doi.org/10.1111/j.1469-8137.2004.01023.x.

23. Peteet D. (2000). Sensitivity and rapidity of vegetational response to abrupt climate change. Proc. Natl. Acad. Sci. 97, 1359-1361. https://doi.org/10.1073/pnas.97.4.1359.

24. Pilon-Smits EAH, Garifullina GF, Abdel-Ghany S, Kato SI, Mihara H, Hale KL, Burkhead JL, Esaki N, Kurihara T, Pilon M. Characterization of a NifS-like chloroplast protein from Arabidopsis. Implications for its role in sulfur and selenium metabolism. Plant Physiol. 2002;130:1309-18. https://doi.org/10.1104/pp.102.010280.

25. Prentice IC, Bartlein PJ, Webb III, T. Vegetation and climate change in eastern North America since the last glacial maximum. Ecology. 1991;72:2038-56. https://doi.org/10.2307/1941558.

26. Reuveni R, Shimoni M, Karchi Z, Kuc J. (1992). Peroxidase activity as a biochemical marker for resistance of muskmelon(Cucumis melo) to Pseudoperonospora cubensis. Phytopathology 82, 749-753. https://10.1094/Phyto-82-749.

27. Saddiqe Z, Naeem I, Maimoona A. A review of the antibacterial activity of Hypericum perforatum L. J Ethnopharmacol. 2010;131:511-21. https://doi.org/10.1016/j.jep.2010.07.034.

28. Samadi S, Khadivzadeh T, Emami A, Moosavi NS, Tafaghodi M, Behnam HR. The effect of Hypericum perforatum on the wound healing and scar of cesarean. J Altern Complement Med. 2010;16:113-7. https://doi.org/10.1089/acm.2009.0317.

29. Singh AP. (2005). Hypericin-A Napthodianthrone from Hypericum perforatum. Ethnobot. Leafl. 2003, 11. https://opensiuc.lib.siu.edu/ebl/vol2003/iss1/11.

30. Singh M, Singh N, Bhandari DK. Interaction of selenium and sulfur on the growth and chemical composition of raya. Soil Sci. 1980;129:238-44.

31. Slattery RA, Ort DR. Photosynthetic energy conversion efficiency: setting a baseline for gauging future improvements in important food and biofuel crops. Plant Physiol. 2015;168:383-92. https://doi.org/10.1104/pp.15.00066.

32. Soleimani A, Valizadeh M, Darvishzadeh R, Alipour H, others, (2017). Evaluation of Yield and Yield Component of Spring Barely Genotypes under Late Season Drought Stress. J. Crop Breed. 9, 105-116. http://jcb.sanru.ac.ir/article-1-881-en.html.

33. Tawaha K, Gharaibeh M, El-Elimat T, Alali FQ. Determination of hypericin and hyperforin content in selected Jordanian Hypericum species. Ind Crops Prod. 2010;32:241-5. https://doi.org/10.1016/j.indcrop.2010.04.017.

34. Thomas FM, Gausling T. Morphological and physiological responses of oak seedlings (Quercus petraea and Q. robur) to moderate drought. Ann For Sci. 2000;57:325-33. https://doi.org/10.1051/forest:2000123.

35. Tozlu E, Cakir A, Kordali S, Tozlu G, Ozer H, Akcin TA. Chemical compositions and insecticidal effects of essential oils isolated from Achillea gypsicola, Satureja hortensis, Origanum acutidens and Hypericum scabrum against broadbean weevil (Bruchus dentipes). Sci Hortic (Amsterdam). 2011;130:9-17. https://doi.org/10.1016/j.scienta.2011.06.019.

36. Uddin MN, Tomita A, Obara M, Yanagihara S, Fukuta Y. Identification of a low tiller gene from a new plant type cultivar in rice (Oryza sativa L.). Breed Sci. 2016;66:790-6. https://doi.org/10.1270/jsbbs.16143.

37. Velikova V, Yordanov I, Edreva A. Oxidative stress and some antioxidant systems in acid rain-treated bean plants: protective role of exogenous polyamines. Plant Sci. 2000;151:59-66. https://doi.org/10.1016/S0168-9452(99)00197-1.

Page $8 / 9$ 
38. Zobayed SMA, Afreen F, Kozai T. Temperature stress can alter the photosynthetic efficiency and secondary metabolite concentrations in St. John's wort. Plant Physiol Biochem. 2005;43:977-84. https://doi.org/10.1016/j.plaphy.2005.07.013.

\section{Figures}

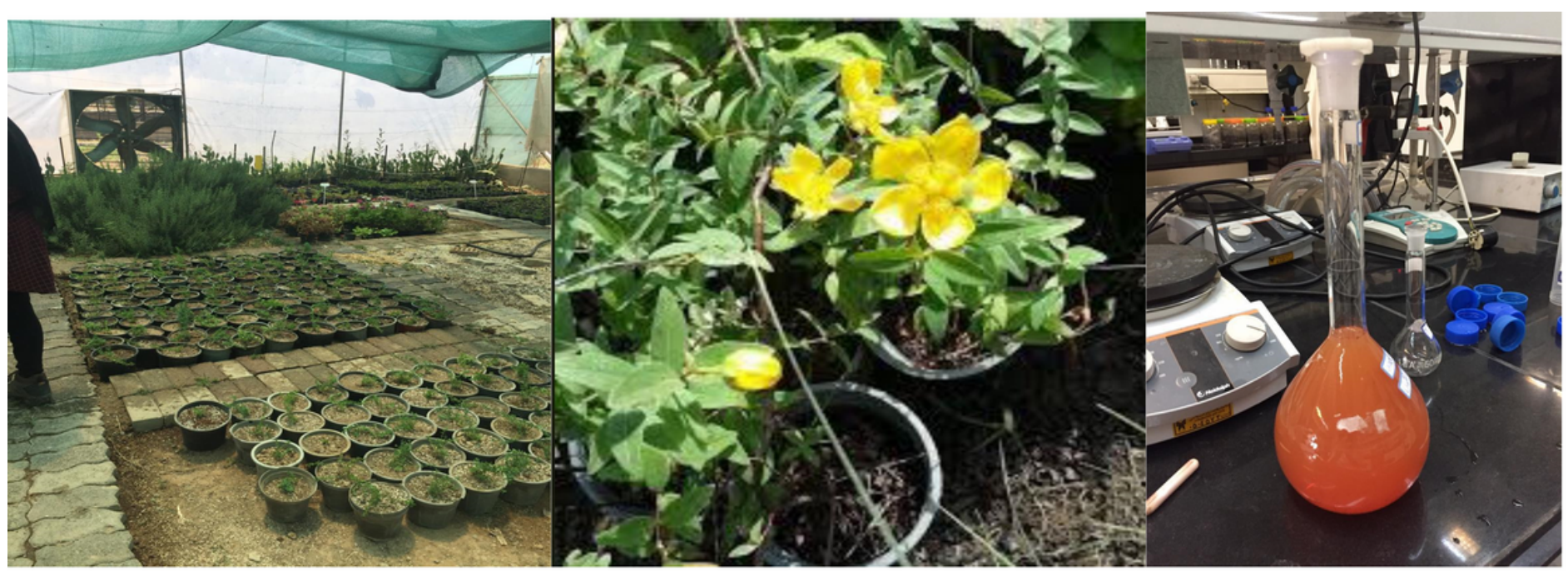

\section{Figure 1}

Pictures of the stages of this research 